\title{
A Framework of Intelligent Evacuation Guidance System for Large Building
}

\author{
Z.Y. Wu \& W.Lv* \& K. Yu \\ Hubei Collaborative Innovation Center for Early Warning and Emergency Response Technology, \\ Wuhan, Hubei, P.R. China \\ China Research Center for Emergency Management, Wuhan University of Technology, Wuhan, \\ Hubei, P.R. China \\ School of Management, Wuhan University of Technology, Wuhan, Hubei, P.R. China
}

\begin{abstract}
KEYWORD: Evacuation; Guidance; Video; RFID
ABSTRACT: When a disaster occurs, it is very difficult for people inside a large building to evacuate safely. We propose a framework of Intelligent Evacuation Guidance System for Large Building (IEGSLB). IEGSLB proposes a new method for the set of evacuation indicators, and fire detection and video surveillance are applied to evacuation guidance, considering the effect of changes in road conditions and population density for dynamic adjustment of evacuation indicators. The mobile terminal communication and RFID system realize collaboration of safety and dangerous routes, minimizing rescue time and saving rescue costs to achieve rescue optimization.
\end{abstract}

\section{INTRODUCTION}

In recent years, along with the process of urbanization, more and more high, large, innovative buildings and public places in design concept came into being. Compared with the traditional architectures, these buildings are different in terms of the use of multiple functions, spatial pattern, structure, materials, facilities, such as shopping malls, railway stations, subways, stadiums, etc. Once a disaster (such as fire, stampede) occurs, not only cause huge damage to property, but also cause massive casualties in more serious cases. Safe evacuation is an important way to protect the safety of personnel when disasters occur in large buildings. However, a large number of disaster cases show that in case of emergency, due to high population density, and most people unfamiliar to the environment, as well as factors such as psychological panic, if there are not proper instructions, diversion measures and unobstructed evacuation facilities in public buildings, overcrowding and even stampede often occur in the exit, stairs and other architectural bottleneck, severely reducing the evacuation efficiency, and easily resulting in heavy casualties. Therefore, when a disaster occurs or even before, how to effectively guide the staff to leave the dangerous area as soon as possible, become a key issue of improving evacuation efficiency, ensuring safe evacuation.

Recently, many works has focused on intelligent evacuation system, especially about fast and effective evacuation in buildings or large public places. Ishii proposes a method to realize evacuation guide in case of power failure. In this system, passive RFID tags are put on walls of the building. Each user has a mobile terminal equipped with an RFID reader/writer. By referring to passive RFID tags which the user touched, the mobile identifies safe routes using the movement of people who have evacuated safely. Reza proposes a method of a network covering algorithm to estimate the position of the electronic tag, and this method is applied to study evacuation. Chen study how apply the RFID technology to the pedestrian movement experiments, besides, obtain a new method to extract the pedestrian movement parameters quickly. Zhang explains the main idea of developing intelligent building evacuation system. There are three main sections in the system, including front information collection, system control module and emergency lighting and evacuation instructions. Sun has also done some related research. According to the fire risk analysis of the building, he divided different fire risk areas. By means of the features of the building fires and the people injury degree, two dangerous states were determined. Although building evacuation system has been stud- 
ied for many years, it just stays in the stage of improvement for fire protection system or simple personnel positioning. In this paper, it is aimed to achieve fast and effective evacuation of personnel, and the linkage of fire protection system, security system and evacuation system, combining multiple technologies in large buildings after a disaster.

This paper is structured as follows. Firstly, summarizes the shortcomings of current evacuation system, and find the problems in actual use. Secondly, introduces the system configuration and principles, at the same time, explains the functions of four subsystems in detail. Finally, a summary concludes the paper.

\section{MOTIVATION}

Fire protection system is an integral part in modern buildings. With the improvement of intelligence in modern buildings, people's safety awareness increasing, there are increasingly high requirements for building fire protection systems. Evacuation safety is one of the important goals in the architecture design of disaster prevention security; therefore, evacuation guidance system plays an important role in large buildings. Currently, evacuation guidance system for large buildings is basically a fixed direction in the traditional evacuation instructions, but a few use a more intelligent dynamic evacuation system, according to the principle of "the Nearest Evacuation" in evacuation. According to the application of these systems, there are still some practical problems have not been solved, mainly in the following aspects:

\section{(1) Limited detecting}

Traditional evacuation guidance system is linked with fire protection system, but failed to link with the security system in construction. Traditional evacuation guidance system can only determine the fire area through the smoke detector and other fire sensing device. It's difficult to detect specific disasters and accidents, or derivative disasters. For example, people cannot enter the fire area when fires happen. At the same time, the channel may be blocked or safe exit door is closed in the evacuation process. These circumstances cannot be detected through the traditional evacuation guidance systems. Evacuation routes which the traditional evacuation guidance systems generate will make it possible to take people to the danger zone, which cannot effectively achieve safe evacuation, and guarantee the safety of personnel to the maximum extent.

(2) Lack of dynamic update

Traditional evacuation indicator has guide function in normal state, in the case of disasters, which the direction of indicator remains fixed, and does not consider the specific circumstances of the building. For example, if a crowded condition occurs in the building channel, the traditional evacuation guidance systems cannot monitor this situation. When people are still evacuated in accordance with the indicator line of defense, it will exacerbate congestion and reduce evacuation efficiency, causing more casualties.

(3) Cannot produce personalized evacuation route

People have a strong herding in the event of a disaster. Traditional evacuation guidance systems can generate evacuation routes for general cases based on the principle of "the Nearest Evacuation ", which easily lead to congestion phenomenon in the evacuation paths.

Overall, the evacuation issue involves many complex factors, such as the mutual influence of people each other, people and structures, people and fire smoke and other environment, people and evacuation guidance system, etc. But the existing evacuation guidance system only stays in the level of simple interaction with fire protection system. For fire and other emergencies, fire protection system can only detect smoke concentration, temperature, flame and other fire signals through smoke detector, temperature sensor and other sensing devices, until the alarm generates a signal and the evacuation system responds to disasters, making the direction of evacuation indicators shift. If we just use the principle of "the Nearest Evacuation", it's unable to monitor the state of security gate, road congestion and damage, and give dynamic feedback. Therefore, it has very important significance to propose and develop a new, intelligent evacuation guidance system for solving problems that the current evacuation system cannot achieve. 


\section{INTELLIGENT EVACUATION GUIDANCE SYSTEM}

\section{Intelligent Evacuation Guidance System}

In order to achieve the target of combining evacuation guidance and assisting rescue, intelligent evacuation guidance system for large building integrate fire alarm, intelligent video recognition and RFID-based networking technology, achieving a linkage in fire systems, security systems and evacuation system, which is divided into five subsystems, and specific system configuration is shown in Fig.1.

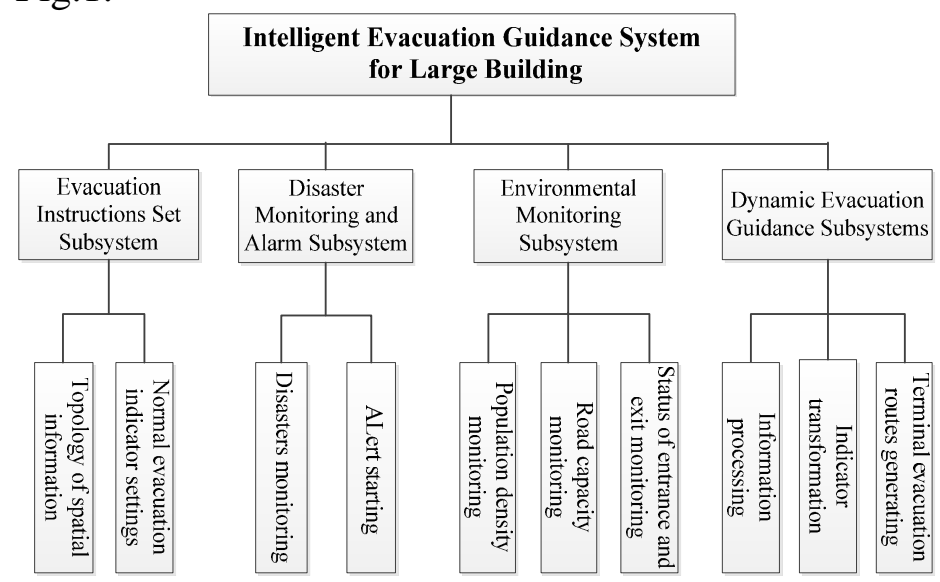

Figure 1. Structure of Intelligent Evacuation Guidance System for Large Building

Evacuation Instructions Set Subsystem (EISS): According to the principle of "the Nearest Evacuation" and the quantity and distribution of exit, set the position and the initial indication orientation of evacuation indicators, making evacuation indicators evenly and reasonably toward the nearest safe exits.

Disaster Monitoring and Alarm Subsystem (DMAS): When confronted with fires and other disasters, the collected and related hazards signals are fed back to the central control room, and then program algorithm begin to assess the current evacuation path, confirming whether the direction of evacuation indicator set according to "the Nearest Evacuation" is reasonable.

Environmental Monitoring Subsystem (EMS): Collect population density information, road capacity and export security status information, and relevant results are fed back to the central control room, auxiliary making real-time calculation and assessment.

Dynamic Evacuation Guidance Subsystems (DEGS): According to the results of the calculation and assessment, dynamic evacuation guidance subsystem adjusts the direction of evacuation indicators, achieving changes from "the Nearest Evacuation" to "Safe Evacuation" and "Efficient Evacuation".

Fig.2 shows the specific operational processes of intelligent evacuation guidance system for large building.

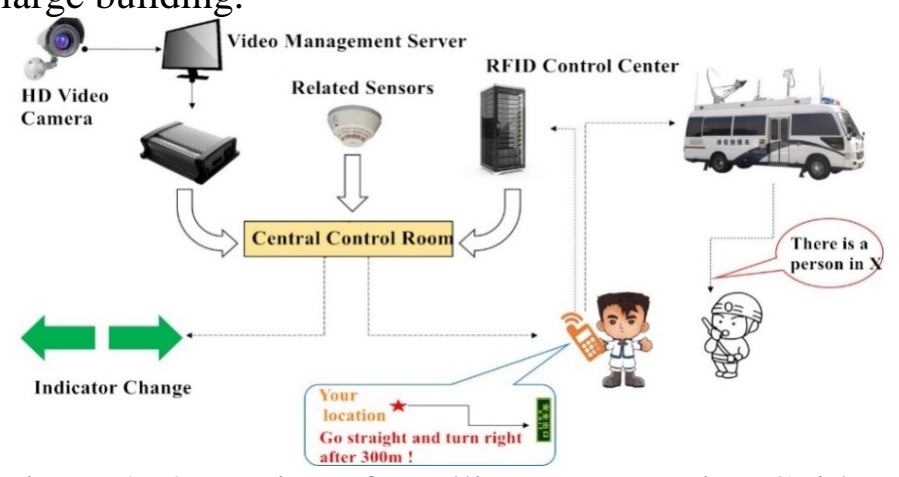

Figure 2. Operation of Intelligent Evacuation Guidance System for Large Building

Evacuation Instructions Set Subsystem

Evacuation instructions set subsystem transform building spatial information into topological diagrams, and further combining with relevant fire regulations, install evacuation lights and the re- 
quired installation of various fire detectors in the building. Setup Requirements: In the absence of the casualty normal circumstances, evacuation indicator point to the nearest safe exit. Setting steps are as follows:

To guide people from their current position to an exit in the building, the terminal must have detailed information on the construction of the building. We propose to represent the construction of the building as a route graph. Import building information into the subsystem, the subsystem will transform flat space topology information into a topology of tree structure. Each node represents a safe outlet (the location of security exit or stairs), each path represents a channel in the building. Path weight represents current difficulty of reaching safe exits successfully passing through this route. Using the theory of optimal path, traverse each node in the tree graph, and to find the optimal path, and to convert the figure of the optimal path into reality map of the route. For example, Fig.3a and Fig. $3 b$ show how to represent path routes in a building as a route graph. Routes are described as a straight line graph. We link them with existing plot such as a room gateway or a stair.

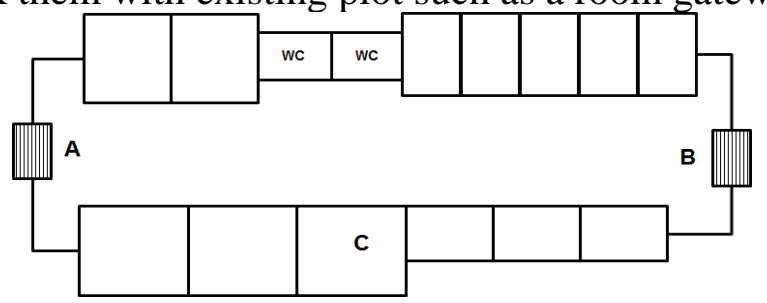

Figure3a. Space structure map of a floor

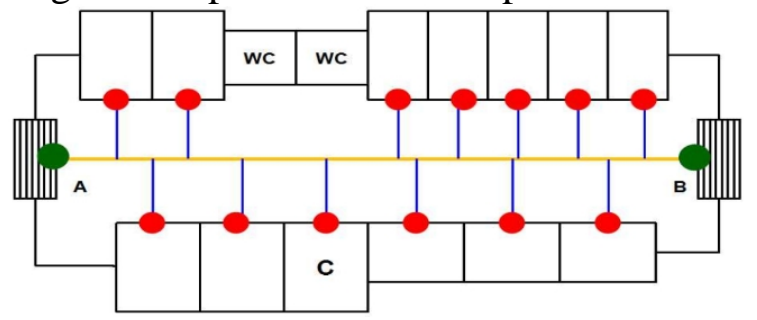

Figure3b. Making a graph from a floor map

In the route graph, each edge of the route graph is converted into a branch of a tree graph, and destinations such as stairs and exits are converted into a root of each tree. The root of a destination is linked nearby the branch by turns. We assume that all tree graphs for each destination are previously made. Tree graphs for destination A and B in Fig.3a and Fig.3b are represented as shown in Fig.4.

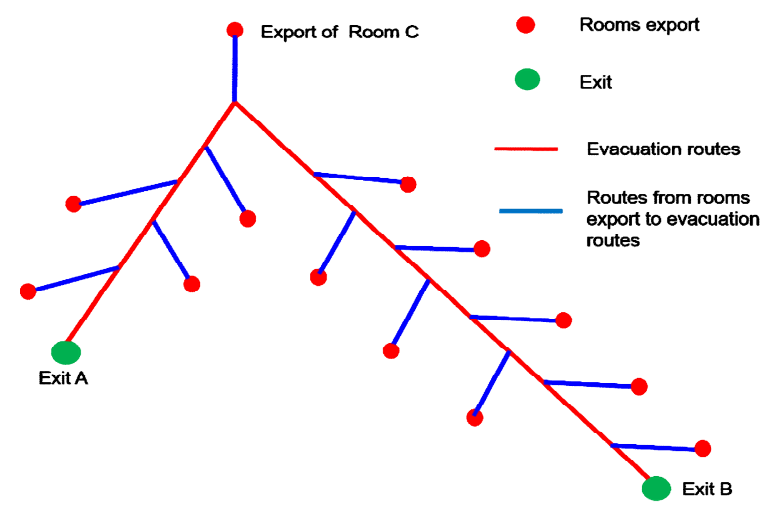

\section{Disaster Monitoring and Alarm Subsystem}

Figure4. Tree structured graph

For all types of disasters faced by large buildings, such as fires, earthquakes, etc. use fire protection system which has been installed in large buildings to monitor the changes in temperature and atmospheric concentration caused by various types of disasters and accidents. Disaster monitoring and alarm subsystem consists of two parts, as follows:

(1) Disasters monitoring 
Install a variety of fire detectors in large buildings according to relevant laws and regulations. Using one or more of smoke detectors, thermal detectors, sensitive detectors, combustible gas detectors and hybrid detectors, get the position, type, intensity of disasters and temperature and visibility changes caused by disasters.

(2) Alert starting

Disasters and accidents occurred in buildings often lead to fires, therefore, fire monitoring is an important task for disaster monitoring and alarm subsystems, which can be used as an important indicator of whether a disaster or accident in the building. When fire detectors detecting a fire, the information is transmitted to the fire alarm control, and alarm system is activated, and then receive, display and transmit a fire alarm signal and shows the location of the fire, time, etc., to help people to discover the fire and take effective measures to put out the initial fire, minimizing loss of life and property caused by fires. In general, automatic fire alarm system and automatic sprinkler system, fire hydrant system, smoke control system, ventilation system, air conditioning system, fire doors, fire shutter, and other related equipment is linked, we can send out the instruction and start the appropriate device automatically or manually.

In the subsystem, we assume that the standard fire hazard, as follows: When the smoke layer interface above the human eye characteristic height $(1.5 \mathrm{~m})$, and the flue gas temperature exceeds $180^{\circ} \mathrm{C}$, it can pose a threat to people; If the smoke layer interface height lower than the human eye characteristics, when $\mathrm{CO}$ concentration exceeds $0.25 \%$ or the flue gas temperature exceeds $115^{\circ} \mathrm{C}$, it will pose a danger to people. When one of these dangerous conditions are satisfied, it is determined that a dangerous state, and then start the alarm system.

\section{Environmental Monitoring Subsystem}

By the impact of disasters and accidents, road conditions and evacuation exit status in the building will have dynamic changes over time. Evacuation density within a region of the case will affect the evacuation efficiency. To ensure the efficient evacuation, environmental monitoring subsystem can achieve the following functions:

(1) Population density monitoring

Monitor population density through video monitors, and the video information is transmitted to the digital video servers to process, and then get the digital information, identifying evacuees density within the monitoring range, and finally communicated to central control room, so that the subsystem control the direction of evacuation indicator, at the same time, send evacuation routes to mobile terminals in evacuees hands.

(2) Road capacity monitoring

Monitor the changes of road capacity caused by the disaster by video monitors. Determine whether the path is suitable as a safe evacuation routes according to the collected video information, such as roads blocked by objects, long light dimmed, the video signal interruption, etc.

(3) Status of entrance and exit monitoring

Using RFID-based technology, we install electronic tags on the door and readers on the building wall. And we can determine the doors' status of the entrance and exit according whether the reader can read electronic tags on the security door. At the same time, using the video monitors to confirm whether the states of entrance and exit are smooth.

\section{Dynamic Evacuation Guidance Subsystems}

Emergency evacuation is a dynamic, complex process of special scenarios under disaster conditions, involving the spread and development of disaster evacuation route selection, optimization of individual, dynamic changes in road capacity, and restructuring and other road network coupling. In practice, the influence of disasters on the road network continues to change over time, causing road capacity and reliability of some roads reduced, or even roads are cut off, which led to the evacuation of the road network in the path to change.

DEGS make dynamic adjustments to evacuation indicators according to disaster development status provided by DMAS and population density information provided by EMS. In the DEGS, evacuation indicators in building are controlled by the central control room, with two-way indicator. It not only can be automatically adjusted according to computer logic circuits, but also for man- 
ual adjustments. Direction adjustment of evacuation indicator is mainly to comply with the following strategies in DEGS:

$a$. When exits cannot be used due to disasters, evacuation indicators originally directed to the exits adjust the pointing direction, pointing to the direction away from the exits;

$b$. When a disaster occurs in front of evacuation indicators a certain range, the system automatically adjusts the direction of evacuation indicators, and abandon this evacuation routes;

$c$. Try to avoid excessively high density of population in the safe exit area, so as to ensure the safety of evacuation;

$d$. Try to balance the evacuation time of every exit, so that the total evacuation time reduced, improving evacuation efficiency.

According to the above evacuation strategies, the subsystem can achieve active evacuation and passive evacuation, as follows:

(1)Active evacuation: The radio frequency modules are embedded in mobile terminals, so that the handheld mobile terminals having a radio frequency identification function. In the evacuation process, reader embedded in the mobile terminal send radio frequency signal and read electronic tag attached to the wall. It can match the actual realization of the space with the map through different reader numbers. The reader embedded in the mobile terminal which is carried by personnel can read electronic tags to locate staff position, and then the central control room can send evacuation routes to handheld mobile terminal.

(2)Passive evacuation: It can monitor corridor condition, population density and other information based video surveillance, fire alarm systems, and then that information is fed back to a central control room, dynamically adjusting the direction of evacuation indicators.

For personnel location, determine traffic information within a certain range of its front according to RFID feedback information. When there are serious buildings collapse or congestion, adjust the direction of evacuation indicators and point to other security exits according to the optimal path. Using video monitoring equipment installed at each exit, analyze the population density of each evacuation region. Determine whether the region will cause traffic congestion according to the population density, and the information is fed back to the central control room, and then make dynamic adjustment to the direction of evacuation indicators, avoiding evacuees flock to the same evacuation exit, affecting the evacuation efficiency.

The steps of searching optimal evacuation path in disaster are as follows:

$a$. According the actual architectural space information and topology with nodes and arcs, establish topology in which a node represents a safe exit (safety door or stairs) and arcs represent the evacuation path connect exits. Wherein, the actual architectural space information includes length, reliability and capacity of path.

$b$. When a disaster occurs in an area, assess the disaster situation in each path according to the intensity and scope of the disaster, and update length, reliability and capacity of paths; and use neural network algorithms to extract $\mathrm{m}$ feasible paths between the disaster area node $O$ to the destination node $D$ from the evacuation path topology map.

c. Select the optimal path from the $\mathrm{m}$ paths according to the updated length, reliability and capacity of path. For example, in order to ensure evacuees speed and security, choose the comprehensive optimal path as the optimal path from the m path among length, reliability and capacity of path.

\section{CONCLUSIONS}

Compared with the prior technology, intelligent evacuation guidance system proposes a new method for the set of evacuation indicators, and fire detection and video surveillance are applied to evacuation guidance, considering the effect of changes in road conditions and population density for dynamic adjustment of evacuation indicators, to provide more comprehensive information about the accident scene for policy-makers and guidance for evacuation and rescue work after public buildings disasters, minimizing rescue time and saving rescue costs to achieve "Optimization Rescue". 
Combine the fire alarm, intelligent video recognition and RFID technology to achieve the linkage of fire protection system, security system and evacuation system. Traditional evacuation system uses the principle of "the Nearby Evacuation", and only point to the nearest safe exit. The system considers path conditions, the state of entrance and exit, and the population density in the evacuation process. The central control room dynamically adjusts the direction of the evacuation lights according to information collected. Traditional evacuation system has achieved passive evacuation. But this system uses RFID technology to achieve precise positioning of personnel, and then combines traffic information received to generate personal best evacuation routes. At last, evacuation routes are sent to evacuees who own mobile terminals, inducing them to evacuate.

\section{ACKNOWLEDGEMENTS}

This research was supported by Hubei Provincial Natural Science Foundation of China (No. 2015CFB593), the Humanities and Social Sciences Program of the Ministry of Education (No. 15YJCZH039), the Fundamental Research Funds for the Central Universities (WUT: 2015VI011, 2015III036).

\section{REFERENCES}

Ahmed, W.R. \& Teoh, W.Y. \& Kaharudin, D. \& Kim, G.T. \& Kamarul, A.N. 2012. International Journal of Electronics. Deployment of a 3D tag tracking method utilising RFID 99(4):557-573.

Ishii, H. \& Shimakawa, H. 2006. FIT2006. Evacuation Guide System with Visualization of Varying Dangerous Area and Person Movement 3-4.

Ishii, H. \& Harada, F. \& Takada, H. \& Shimakawa, H. 2011. Electrical Engineering. Reassuring evacuation guidance with mobile terminal communication and RFID system in absence of power supply 177(2):19-31.

Kawamura, H. \& Ohuchi., A. Computer Science. Modeling of theme park problem with multiagent for mass user support 3012:48-69.

Zhang, Q. \& Chen, T. \& Lv, X.Z. 2011. Fire Science and Technology. The architecture of intelligent building evacuation system (3): 205-207. 